Obtaining a natural dye from the pitahaya (Hylocereus undatus haworth, britton and rose) from the southern region of the state of Campeche

\title{
Obtención de un colorante natural a partir de la pitahaya (Hylocereus undatus haworth, britton y rose) de la región sur del estado de Campeche
}

Martha Elena Cervantes-Sánchez*, Jorge Luis Huicab-Martínez, José Alfredo García-Vela, Maximiliano Vanoye-Eligio

Instituto Tecnológico Superior de Escárcega. Ingeniería en Industrias Alimentarias. Calle $85 \mathrm{~s} / \mathrm{n}$ entre 10B, Col. Unidad Esfuerzo y Trabajo I, Escárcega. Campeche. Tel: 982 5960603; Tel: 9825960604.

*Corresponding author.

E-mail address: mcervantes_iia@itsescarcega.edu.mx (M. E. Cervantes-Sánchez).

Article history:

Received: 29 November 2016 / Received in revised form: 31 May 2017 / Accepted: 14 June 2017 / Published online: 1 July 2017

https://doi.org/10.29267/mxjb.2017.2.2.65

\begin{abstract}
The pitahaya is a fruit that contains beneficial properties for humans, among which are the pigments used for coloring in the food industry, such as anthocyanins and betaleínas with antioxidant activity. It is important to note that they are not the only beneficial substances for humans that has pitahaya, it also contains thiamine and ascorbic acid. It is for this reason that the objective of this work was the obtaining of a natural colorant from the shell of pitahaya with potential use in the food industry. For the achievement of the work the pitahaya shell was used to obtain the natural dye. The dye was obtained through different techniques, such as dehydration, drying, shredding of the shell, sieving and the use of a centrifuge. The results indicate that the dye obtained from dehydration is part of the group of anthocyanins and that at a ratio of $1 / 10$ is sufficient to give color to fermented beverages.
\end{abstract}

Keywords: Pitahaya, extraction, dye, Campeche.

\section{RESUMEN}

La pitahaya es una fruta que contiene propiedades benéficas para el humano, entre las cuales destacan los pigmentos que se utilizan para la coloración en la industria alimentaria, tales antocianinas y betaleínas con actividad antioxidante. Es importante señalar que no son las únicas sustancias benéficas para el ser humano que tiene la pitahaya, también contiene, tiamina y ácido ascórbico. Es por ello, que objetivo de este trabajo fue la obtención de un 
colorante natural a partir de la cáscara de pitahaya con uso potencial en la industria alimentaria. Para el logro del trabajo se utilizó la cáscara de pitahaya para la obtención del colorante natural. El colorante fue obtenido a través de diferentes técnicas, tales como la deshidratación, secado, trituración de la cáscara, tamizado y el uso de una centrífuga. Los resultados indican que el colorante obtenido a partir de la deshidratación forma parte del grupo de antocianos y que a una relación de 1/10 es suficiente para dar color a bebidas fermentadas.

Palabras clave: Pitahaya, extracción, colorante, Campeche.

\section{INTRODUCCIÓN}

La coloración en los alimentos de origen vegetal y animal es determinante para su comercialización y consumo, ya que el color indica la calidad del alimento (Ortega, 2004). Dicha coloración en los alimentos son producidos de manera natural por diversas sustancias químicas, por lo que Roquero (1995) define que un colorante natural es aquel que se sintetiza y acumula en las células de plantas y animales. Una de las sustancias químicas que da coloración a los alimentos y que ha impactado en la industria alimentaria son las antocianinas y betalaínas, ya que estas presentan colores atractivos (Konczack \& Zhang, 2004). En el caso de la pitahaya, presenta alto contenido de betalaínas, pigmentos que han sido considerados como una alternativa al uso de colorantes artificiales en alimentos (Esquivel \& Araya, 2012), por lo que hoy en día diversos productos que contienen antocianinas y betalaínas de manera natural son de origen vegetal, las cuales están siendo incorporadas a productos alimenticios con la finalidad de dar coloración, por ejemplo el color que tienen las tortillas azules se debe a las antocianinas, las cuales se pueden encontrar a la venta en tiendas departamentales y locales. Sin embargo, es muy común encontrar en el mercado nacional, regional y local productos alimenticios con colorantes sintéticos, los cuales han sido duramente cuestionados en muchos países porque se les ha ligado con el desarrollo de enfermedades (Wang et al., 1997). El color rojo, el cual ha sido muy utilizado a gran escala en la industria alimentaria se ha prohibido, tal es el caso de la suspensión del uso de E-126 Rojo 2G como colorante alimentario (Barros, 2009) o el rojo No. 40 (Garzón, 2008), por lo que los colorantes naturales de origen vegetal tienen la oportunidad de insertarse en el mercado a nivel nacional, tal es el caso de las antocianinas y betaleínas que son una alternativa importante para sustituirlos (Harborne, 1967; Roquero, 1995; Fabbio et al., 2009). En el caso de las antocianinas, estas han sido consumidas por la humanidad desde nuestros antepasados sin ningún efecto perjudicial evidente, por lo que su adición en los alimentos, además de dar coloración, también presenta diversas cualidades benéficas para el ser humano (Caballero, 1995; Wang et al., 1999; Klinger, 2002). Diversos estudios han reportado que el uso de las antocianinas son beneficiosas para la salud del humano, como se ha demostrado en diversos estudios, los cuales evidencias que las antocianinas pueden mejorar la agudeza visual, mostrar actividad antioxidante, atrapar radicales y actuar como agentes quimioprotectores. Las antocianinas también juegan un papel en las propiedades antidiabéticas tales como control de lípidos, secreción de insulina y efectos vasoprotectivos (Ghiselli et al., 1998; Miyazawa et al., 1999; Astrid, 2008; Shipp \& Abdel-Aal, 2010). Es por ello que Roquero \& Martínez, (2010) afirman que el mundo de los colorantes naturales y sus aplicaciones es un campo claramente inter y 
multidisciplinario, que ha vuelto a renacer, teniendo por delante un futuro prometedor y benéfico para la humanidad.

Actualmente la extracción de colorantes naturales de diversas especies vegetales está presentado mayor auge, sin embargo, existen especies del reino vegetal en el sureste mexicano que presentan un gran potencial como colorantes naturales a las cuales no se les ha puesto atención, tal es el caso de la pitahaya (Hylocereus undatus), la cual se produce en el estado de Campeche, que de acuerdo al Servicio de Información Agraria y Pesquera (2009) es el quinto productor de pitahaya, siendo Yucatán el primer productor. Por otro lado, el cultivo de pitahaya se adapta bien a climas cálidos subhúmedos, con lluvias en verano. La planta, en sus primeros años, es muy susceptible a las heladas, se desarrolla en lugares con muy poca agua disponible; a los 2 años de plantada, el cultivo empieza a producir frutos, aunque en pequeñas escalas (Murillo, 2013).

La pitahaya es una planta que pertenece a la familia de las Cactaceae, que se pueden consumir como fruta fresca o procesada como pulpa; sin embargo, resulta importante retomar el estudio de los componentes del mercado, en donde se destaca no sólo por ser una fruta con componentes nutricionales que aportan beneficios a la salud humana, sino también porque suple una demanda latente y su cultivo puede ser una actividad alternativa para productores de zonas con condiciones agroecológicas adversas (García-Barquero \& Quirós-Madrigal, 2010). El uso que se ha detectado para esta fruta en la región es únicamente como bebida fresca, lo que significa que solo se aprovecha la parte comestible del fruto, que solo representa aproximadamente el $55 \%$ del peso total del fruto, siendo el otro $45 \%$ del peso, mientras que la parte restante es cáscara, que se desecha o se utiliza como forraje (Figueroa et al., 2011), por lo que las características biológicas de la fruta representan a esta especie una importante fuente para la extracción de colorantes naturales. Es por ello que la presente investigación tuvo como objetivo principal la extracción de pigmentos como colorantes a partir de la pitahaya que se produce en el estado de Campeche.

\section{MATERIALES Y MÉTODOS}

El estudio se realizó en el municipio de Escárcega, el cual se ubica entre $18^{\circ} 51^{\prime}$ y $18^{\circ} 09^{\prime}$ $\mathrm{N}$ y $90^{\circ} 2^{\prime}$ y $91^{\circ} 33^{\prime} \mathrm{O}$, a $60 \mathrm{~m}$ de altitud y su extensión es $4569.64 \mathrm{~km} 2(8.04 \%$ de la superficie del estado de Campeche). En él desembocan la cuenca Laguna de Términos y otros ríos, y la de Yucatán oeste (Campeche). El clima es cálido sub-húmedo con lluvias en verano, subtipo humedad media (Aw1), con una temperatura promedio anual de $26{ }^{\circ} \mathrm{C}$ (INEGI, 2015), lo que favorece el crecimiento y producción de la pitahaya a diferentes escalas. La obtención de la materia prima se hizo principalmente en el mercado local de la Ciudad de Escárcega y en ejidos aledaños. Una vez obtenida la materia prima se le hizo un pre-tratamiento que consistió en lavarla de manera tradicional para limpiarla de impurezas. Posteriormente se realizó el proceso de extracción del colorante:

\subsection{Proceso en detallado de la extracción del colorante en la pitahaya}

2.1.1. Pelado: la cáscara contiene partes puntiagudas gruesas, las cuales se eliminan para dejar solo la cáscara de la fruta. 
2.1.2. Rebanado: la cáscara de la fruta se rebana en cortes delgados para facilitar el secado.

2.1.3. Deshidratado: las rebanadas se deshidratan por un tiempo de 1:30 para eliminar la humedad de la cáscara.

2.1. 4. Ira Horneada: La cáscara deshidratada se hornea por un tiempo de 30 minutos a una temperatura de $40^{\circ} \mathrm{C}$. Cabe señalar que cada 10 minutos hay que estar moviendo la cáscara con la finalidad de que no se pegue en las charolas, en las cuales fue colocada.

2.1.5. 2 da horneada: la segunda horneada se realiza por un tiempo de 2 horas a $105^{\circ} \mathrm{C}$. La temperatura se aumentó con la finalidad de eliminar en su totalidad la humedad que todavía pudiese encontrar en la cáscara y al igual que en la primera horneada hay que estar moviendo la cáscara cada 10 minutos para que no se pegue en las charolas.

2.1.6. Triturado: una vez estando la cáscara seca en su totalidad, se trituró con una licuadora industrial. Este paso consistió básicamente en observar cada tres minutos el triturado buscando que no queden residuos.

2.1.7. Tamizado: se tamizó lo obtenido del triturado y los grumos obtenidos son triturados nuevamente con la finalidad de aprovechar en su totalidad toda la cáscara.

2.1.8. Mezclado: se realizó con agua purificada en una porción de $500 \mathrm{ml}$ de agua y 15 gramos del polvo obtenido en el tamiz y se homogenizó hasta quedar disuelto por completo el polvo.

2.1.10. Centrifugado: de la mezcla se extrajeron $7 \mathrm{ml}$ con una pipeta y se depositan en los tubos de ensayo, los tubos con $7 \mathrm{ml}$ de la mezcla se centrifugaron por un tiempo de 20 minutos a $2000 \mathrm{rpm}$, después de este tiempo se extrae el sobrenadante de los tubos.

2.1.11. Obtención del colorante: el sobrenadante de los tubos se extrajo con una pipeta y se depositó en frascos para su conservación.

El colorante obtenido fue probado de manera exploratoria en el área de repostería en la elaboración de merengue. (Figura 1) y en bebidas alcohólicas para darle color una relación $1: 10$. 


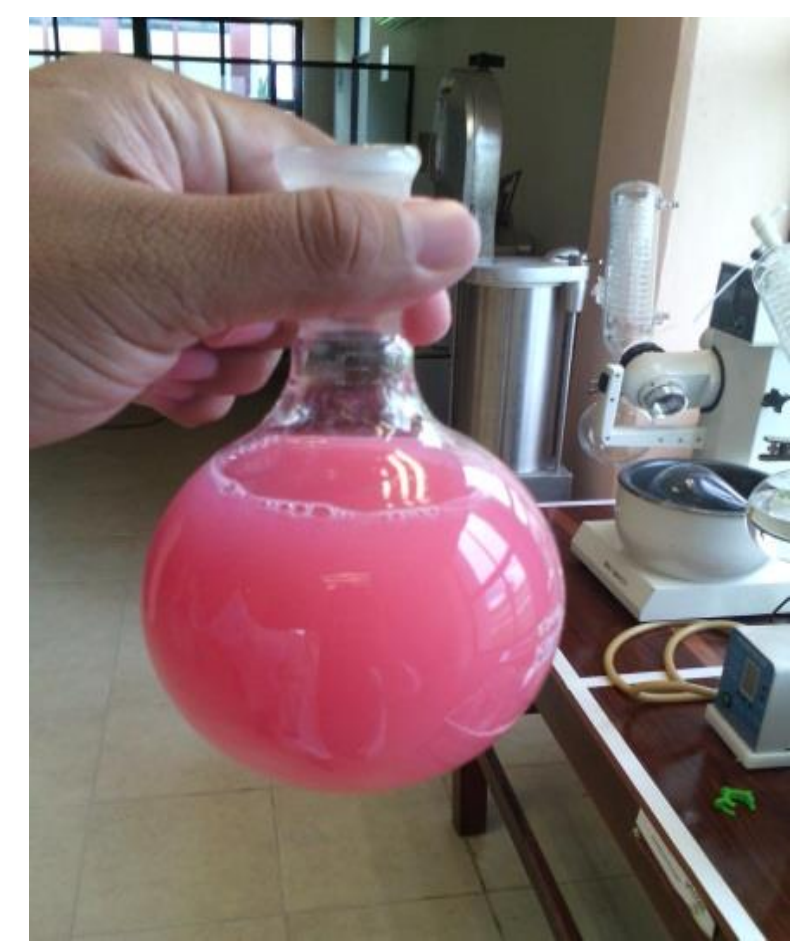

Fig. 1. Colorante extraído de la pitahaya.

\section{RESULTADOS}

La extracción del colorante a partir de la pitahaya se realizó de manera satisfactoria, ya que se logró estandarizar el proceso para su extracción. De acuerdo a la clasificación de Roquero (1995) el colorante extraído forma parte del grupo de antocianos. Los resultados no fueron diferentes en el porcentaje de extracción del colorante. De manera general, por cada 100 gramos de cáscara se logró extraer el 6\% de colorante en forma de polvo (tabla 1).

Tabla 1. Rendimiento que se obtienen en el proceso de extracción del colorante.

Peso inicial de la materia prima

Peso en el deshidratador con un tiempo de 1 hora con 30 minutos

Primera horneada por un tiempo de 30 minutos a $40^{\circ} \mathrm{c}$

Segunda horneada por un tiempo de 2 horas a $105^{\circ} \mathrm{c}$

Peso después del triturado con la licuadora

Peso perdido en el tamizado

Peso neto del polvo
542 grs

182 grs

152 grs

50 grs

32.784 grs

1.984 grs

30.8 grs 
En otros estudios se ha reportado un rendimiento mayor en la extracción de colorantes a partir de otros órganos de las plantas (Abadía et al., 2003), que varían desde el 18\% en frutos y semillas hasta el $29 \%$ en hojas, siendo estas últimas en donde se ha reportado un mayor rendimiento para la extracción de colorantes, sin embargo, es necesario señalar que los resultados expuestos incitan al uso de la cascara de pitahaya, la cual en la región se considera como un desecho agrícola.

Por otra parte la tonalidad del colorante fue un rosa moderado (Figura 1). Este color se obtuvo cuando la fruta se encontraba en un estado fisiológico total de madurez, ya que en estudios anteriores se ha demostrado que cuando los frutos presentan bastante madurez el colorante obtenido es mayor y vistoso, además de que es posible que se obtenga una coloración diferente y de mejor calidad, si se hacen pruebas con las hojas y tallos de estas especies (Chalá et al., 2003).

\section{DISCUSIÓN}

En la investigación se obtuvo un colorante natural en forma de polvo a partir de la cáscara de pitahaya considerada como desecho agrícola en la región. El colorante obtenido se considera fuente natural de antocianinas con capacidad antioxidantes, las cuales tienen una aplicación potencial en alimentos o la industria farmacéutica (Figueroa et al., 2011). Asimismo, los frutos del género Hylocereus se han propuestos utilizarlos en la industria alimentaria para la extracción de colorantes naturales (Stintzing et al., 2003). Sin embargo, la producción y uso de la pitahaya hoy día se limita solo al uso de la pulpa, ya que esta tiene una gran demanda en la industria alimentaria en los Estados Unidos y Europa (OrtizHernández, 1999), de la misma forma ocurre a nivel local y regional, en donde la pitahaya solo es aprovechada por temporada, abundando en los mercados locales de cada lugar en la península de Yucatán. En el municipio de Escárcega, así como en el estado de Campeche la venta de la pitahaya es en forma de fruto, o en su caso en forma de agua fresca, la cual es envasada en botellas de plástico de manera artesanal (Huicab, 2016). Es muy importante señalar que la extracción del colorante a partir de la pitahaya contiene antocianinas, pero no es la única especie de la cual se obtienen las antocianinas, la remolacha roja (Beta vulgaris ssp. vulgaris) es una de las especies de la cual se obtienen antocianinas, sin embargo son poco utilizadas por su inestabilidad a los cambios de $\mathrm{pH}$ y de temperatura, por lo que las antocianinas que se obtienen de la pitahaya son más estables frentes a estos dos factores (Esquivel \& Araya, 2012).

Por otro lado, de acuerdo al proceso aplicado para la obtención de un colorante natural a partir de la pitahaya se observó que de cada 100 gramos de cáscara se obtuvo un $6 \%$ (6 gramos) de colorante natural en forma de polvo, estas valores son similares a los mencionados por otros autores (Abadía et al., 2003), por lo que su uso en la industria alimentaria se recomienda, ya que las cantidades que se obtienen de la cáscara de pitahaya son adecuadas para su uso, de la misma manera Figueroa et al., (2011) obtuvo resultados similares en su trabajo de investigación, en el cual muestra que la cáscara de pitahaya (Hylocereus undatus) es una fuente natural de antocianinas con capacidad antioxidantes, las cuales tendrían una aplicación potencial en alimentos o la industria farmacéutica, por su parte, Esquivel \& Araya (2012) mencionan que la composición química de la pitahaya tienen betalaínas, el cual se considera un colorante natural importante y de la misma forma 
demostraron las propiedades antioxidantes y probióticas que presenta la pitahaya por lo que estos frutos son una alternativa muy importante para la obtención de productos alimenticios con propiedades nutracéuticas o productos farmacéuticos, entre otras propiedades, asimismo Rojas et al. (2015) comparten los resultados, estos autores proponen que los habitantes de Acaquizápan se beneficien con nuevas fuentes de empleos e ingresos monetarios mediante la extracción de colorantes naturales de la cáscara de la pitahaya, ya que presenta una amplia gama de colores de uso seguro en el área de alimentos en primera instancia, así como en las industrias cosmética, farmacéutica y textil.

\section{AGRADECIMIENTOS}

Agradecemos las facilidades y apoyos otorgados para la realización del Proyecto "Aprovechamiento integral de la pitahaya (Hylocereus undatus) para la elaboración de una bebida en presentación tipo vino conservando las características sensoriales de la fruta", el cual fue aprobado ante TecNM con clave 258.15-PD en Octubre de 2015.

Al Laboratorio de Ingeniería en Industrias Alimentarias del Instituto Tecnológico Superior de Escárcega por el espacio brindado.

\section{CONFLICTO DE INTERESES}

Los autores que participaron en el trabajo de investigación para la elaboración y redacción del escrito titulado "OBTENCIÓN DE UN COLORANTE NATURAL A PARTIR DE LA PITAHAYA (Hylocereus undatus Haworth, Britton y Rose) DE LA REGIÓN SUR DEL ESTADO DE CAMPECHE", Martha Elena Cervantes-Sánchez, Jorge Luis HuicabMartínez, José Alfredo García-Vela y Maximiliano Vanoye-Eligio manifiestan que no existe conflictos de intereses de ninguna índole y de la misma forma lo manifiesta la institución a la que se encuentran adscritos.

\section{REFERENCIAS}

Abadía B. D., Castro R. A. \& Pino B. N. 2003. Una aproximación al uso tradicional de las especies vegetales colorantes en el municipio de Quibdó, Chocó. En memorias: VI Seminario Internacional del Medio Ambiente y Desarrollo Sostenible. Octubre 8, 9 y 10 de 2003. Bogota, Colombia.

Astrid G. G. 2008. Las antocianinas como colorantes naturales y compuestos bioactivos: revisión. Acta Biológica Colombiana, 13(3), 27-36.

Barros, C. 2009. Los aditivos en la alimentación de los españoles y la legislación que regula su autorización y uso. Editorial Visión Libros.

Caballero R. 1995. La etnoboranica en las comunidades negras e indrgenas del delta del rio Patfa. Edit. ABYA- YALA.Ecuador. 
Chalá W. P., Guerrero J. E., Rivas A. C., Castro A. A., Palacios J. A. \& Castro A. 2003. Extracción artesanal de colorantes naturales, una alternativa de aprovechamiento de la diversidad biológica del chocó, Colombia. Acta Biológica Colombiana, 8(2), 95-98.

Esquivel P. \& Araya Q. Y. 2012. Características del fruto de la pitahaya (Hylocereus sp.) y su potencial de uso en la industria alimentaria. Revista Venezolana de Ciencia y Tecnología de Alimentos. 3 (1): 113-129.

Fabbio F. A., Hilgert N. I. \& Lambaré D. A. 2009. Los tintes naturales de Los Toldos y alrededores. $1^{\circ}$ Ed. CYTED-RISAPRET, San Salvador de Jujuy, 90 pp.

Figueroa R., Tamayo J., González S., Moreno G. \& Vargas L. 2011. Actividad antioxidante de antocianinas presentes en cáscara de pitahaya (Hylocereus undatus). Revista Iberoamericana de Tecnología Postcosecha, 12(1): 44-50.

García-Barquero M. E. \& Quirós-Madrigal O. 2010. Análisis del comportamiento de mercado de la pitahaya (Hylocereus Undatus) en Costa Rica. Tecnología en Marcha, 23(2), $14-24$.

Garzón, G. A. 2008. Las antocianinas como colorantes naturales y compuestos bioactivos: revisión. Acta Biológica Colombiana, 13(3), 27-36.

Ghiselli A., Nardini M., Baldi A. \& Scaccini C. 1998. Antioxidant activity of different phenolic fractions separted from an Italian red wine. Journal Agricultural and Food Chemistry, 46(2),361-367.

Harborne J. B. 1967. The Anthocyanin pigments. En: Comparative biochemistry of the flavonoids. New York: Academic Press. p.1-30.

Huicab M. J. L. 2016. Aprovechamiento integral de la fruta pitahaya (Hylocereus undatus) para la elaboración de una bebida en presentación tipo vino. Tesis de Licenciatura. Instituto Tecnológico Superior de Escárcega. 68 pp.

INEGI. Instituto Nacional de Estadística Geografía e Informática. 2015. Disponible en http://www.inegi. gob.mx/geo/default.asp?e=04 (consultado Diciembre de 2015).

Klinger W., Pinzón A., Pachón M., Rojas L. \& Aragón J. 2002. Estudio de las especies promisorias productoras de colorantes en el trapecio amaz6nico. Centro de investigación y Desarrollo Científico, Universidad Distrital Francisco Jose de Caldas. Bogota.

Konczack I. \& Zhang W. 2004. Anthocyanins-more than Nature's Colours. Journal Biomedical and Biotechnology, 5:239-240.

Miyazawa T., Nakagawa K., Kudo M., Muraishi K. \& Someya K. 1999. Direct intestinal absorption of red fruit anthocyanins, cyaniding-3-glucoside and cyaniding-3,5-diglucoside, into tracts and humans. Journal Agricultural and Food Chemistry, 47, 1083-1091. 
Murillo P. C. W. 2013. Proyecto viable para la exportación de pitahaya amarilla con destino a Alemania, 41(20), 205-250.

Ortega V. 2004. Estudio Comparativo en el uso de colorantes naturales y sintéticos en alimentos, desde el punto de vista funcional y toxicológico (Doctoral dissertation, Tesis]. Chile: Ingeniería de alimentos. Facultad de Ciencias Agrarias. Universidad Austral de Chile).s

Ortiz-Hernández Y. D. 1999. Pitahaya, a new crop for Mexico. México: Limusa.

Rojas P. P. C., Gijón C. A. S. \& Luis G. Y. J. 2015. Producción de pitaya como alternativa de desarrollo local en Acaquizápan, Santiago Chazumba, Oaxaca. $20^{\circ}$ Encuentro Nacional sobre Desarrollo Regional en México. Cuernavaca, Morelos del 17 al 20 de noviembre de 2015. AMECIDER - CRIM, UNAM. 20 pp.

Roquero A. 1995. Colores y colorantes de América. Anales del Museo de América, 3: 145160.

Roquero A. \& Martínez V. F. M. 2010. Presente y futuro de los colorantes naturales.

Shipp J. y Abdel-Aal S. M. 2010. Food Applications and Physiological Effects of Anthocyanins as Functional Food Ingredients. The Open Food Science Journal, 4:7-22.

Stintzing F. C., Schieber A. \& Carle R. 2003. Evaluation of colour properties and chemical quality parameters of cactus juices. European Food Research and Technology. 216(4):303311.

Wang F., Couture R., Do C. B., Pham T. Q. \& Tong V. H. 1997. Properties of anthocyanins from grape cell culture. J Food Sci, 62(2):246-248.

Wang H., Fair M. G., Chang Y. C., Booren A. M., Gray J. I. \& Dewitt D. L. 1999. Antioxidante and anti-inflammatory activities of anthocyanins and their aglicons, cyanidin, froma tart cherries. J Nat Prod, 62(2):294-296. 